\title{
OBSERVATIONS OF AEROSOL SPATIAL DISTRIBUTION AND EMISSIONS IN NEW YORK CITY USING A SCANNING MICRO PULSE LIDAR
}

\author{
Adrian Diaz Fortich*2 ${ }^{2}$ Victor Dominguez ${ }^{2}$, Yonghua Wu ${ }^{1}$, Barry Gross ${ }^{1}$, Fred Moshary ${ }^{1}$ \\ ${ }^{1}$ NOAA Center for Earth System Science and Remote Sensing Technology \\ Electrical Engineering Department, City College of New York, New York, NY 10031, USA \\ ${ }^{2}$ NOAA EPP Earth System Science and Technology Fellow \\ *Email: adiaz001@citymail.cuny.edu
}

\begin{abstract}
In order to better understand the behavior of particulate pollution and atmospheric dynamics in New York City, it is of great importance to analyze the spatial distribution of aerosols. A scanning lidar system allows for horizontal range-resolved observations of aerosol backscatter with high space and time resolution. A challenge to analyzing the lidar returns is to disentangle extinction over the range of the observations to retrieve the backscatter coefficient with distance. This work presents horizontal measurements taken with a scanning eye-safe Micro Pulse Lidar in New York City. The measurements are analyzed using the Slope Method to get an estimate of the range-resolved aerosol backscatter coefficient. The results are presented as backscatter coefficient maps that display the aerosol spatial distribution within the field of view of the scanning pattern deployed. These observations clearly resolve aerosol dynamics and emission sources within the urban areas.
\end{abstract}

\section{INTRODUCTION}

Particulate pollution is a great concern in densely populated urban areas, such as New York City, as studies have shown that high exposure to fine particulate matter (particulates with a diameter of less than $2.5 \mu \mathrm{m}$, also known as $\mathrm{PM}_{2.5}$ ) can be associated with adverse health effects on human respiratory and cardiovascular systems [1-2]. Lidar imaging of the spatial distribution of aerosols can shed light on particulate pollution and atmospheric dynamics. Vertical distribution of aerosols above the CCNY campus have been observed using a Raman-Mie lidar and ceilometer and have produced important studies on particulate pollution and plume transport [3-5]. However, vertically pointing instruments are unable to obtain multi- dimensional views of the atmosphere, and aerosol dynamics across the cityscape, hence scanning lidars are needed.

Previous implementations of scanning lidars at other locations have combined transmitter sources in different regions of the spectrum with different lidar techniques, some of them include elasticbackscatter lidar in the UV [6], differential absorption lidar in the IR [7] and Raman-shifted and polarization lidar in the IR [8-9]. Scanning lidars are required to perform rapid scans with moderate range, which can be achieved with high pulse-energy sources that allow measurements with reduced averaging time. However, these are usually not eye-safe and must be operated as established by the strict guidelines set forth by the US Federal Aviation Administration, and American National Standards Institute (ANSI) guidelines for Safe Use of Lasers Outdoors [10]. On the other hand, micro-pulse lidars (MPL) are simple, low cost and more importantly, meet the necessary eye-safety requirements for scanning. MPL consists of a transmitter source with $\mathrm{kHz}$ repetition rate and pulse energy on the order of micro Joules. The energy transmitted by this source meets the eye-safety requirements at all ranges when appropriately expanded in the beam size. Moreover, the receiver employs an effective photon counting detection using a compact avalanche photo diode detector [11]. Currently, MPLs are used in NASA's Micro-Pulse Lidar Network (MPLNET), which continuously measures boundary layer heights as well as aerosol and cloud vertical structure from multiple sites around the globe [12].

In this study, a commercial scanning eye-safe miniMPL is used to perform horizontal measurements from which backscatter coefficient maps are derived. These maps display the aerosol spatial 
distribution and emission sources present within the field of view of the scanning pattern used.

\section{METHODOLOGY}

The instrument used, the Mini-MPL by Sigma Space Corp., is a scanning eye-safe elasticbackscatter lidar, located on top of the Grove School of Engineering at City College of the City University of New York.

\begin{tabular}{|l|l|}
\hline \multicolumn{2}{|l|}{ Transmitter } \\
\hline Wavelength & $532 \mathrm{~nm}$ \\
\hline Repetition rate & $2500 \mathrm{~Hz}$ \\
\hline Energy & $4 \mu \mathrm{J}$ \\
\hline Receiver & \multicolumn{2}{|l|}{} \\
\hline Diameter & $8 \mathrm{~cm}$ \\
\hline Field of view & $220 \mu \mathrm{rad}$ \\
\hline Detector & APD photon counting \\
\hline Range resolution & $5 / 15 / 30 / 75 \mathrm{~m}$ \\
\hline
\end{tabular}

Table 1. Mini-MPL specifications.

As defined by Campbell et al. [13], MPL measured raw photon counts take the form:

$n(r)=\frac{\frac{C E O(r) \beta(r) T(r)^{2}}{r^{2}}+n_{b}+n_{a p}(r)}{D[n(r)]}$

Where $n(r)$ equals the measured signal return in photoelectron counts per microsecond at range $r$.C represents a dimensional system calibration constant. $E$ is the transmitted laser pulse energy. $O(r)$ is the overlap correction as a function of range caused by the field of view of the transceiver system. $\beta(r)$ is the backscatter coefficient due to all types of atmospheric scattering. $T(r)$ is the atmospheric transmittance. $n_{b}$ is the background contribution from ambient light. $n_{a p}(r)$ is the contribution from afterpulse, and $D[n(r)]$ is the detector deadtime correction factor as a function of raw counts. Rearranging the terms:

$N(r)=C \beta(r) T(r)^{2}=\frac{\left\{n(r) D[n(r)]-n_{b}-n_{a p}(r)\right\} r^{2}}{E O(r)}$

In this new form, $N(r)$ represents the Normalized Relative Backscatter (NRB), which is the primary product of MPL as it includes corrections for the different artifacts present in the raw measurements. The NRB is a quantity proportional to the attenuated backscatter as it is directly affected by the atmospheric transmittance term $T(r)$. This implies that the NRB values on the far end will tend to be small due to path attenuation. In order to correct for this and have a better representation of the aerosol distribution, it is necessary to further operate on the NRB values looking to separate the atmospheric transmittance term from the backscatter coefficient.

$T(r)=e^{-\int_{0}^{r} \sigma\left(r^{\prime}\right) d r^{\prime}}$

The atmospheric transmittance is defined in terms of the extinction coefficient, $\sigma(r)$ (Equation 3). Therefore, it is necessary to know the extinction coefficient at all ranges in order to calculate the atmospheric transmittance. The slope method of inversion defines the extinction coefficient for a homogeneous atmosphere, $\sigma_{\text {hom }}$, in terms of the signal slope, as follows [14]:

$\sigma_{\text {hom }}=-\frac{1}{2} \frac{d \ln [N(r)]}{d r}$

The accuracy of this method increases with increasing aerosol concentration, favoring its application to the lidar measurements conducted in the boundary layer where the highest aerosol concentrations are usually observed [15]. Typically, the slope of the least squares straight line fit to $\ln [N(r)]$ over any interval where it appears to be nearly a straight line is used as the best estimate of $\frac{d \ln [N(r)]}{d r}$. In other words, the slope method can be applied to determine the extinction coefficient only in certain parts of the signal. Since it is necessary to calculate the atmospheric transmittance $T(r)$ at all ranges, the extinction coefficient obtained using the slope method, $\sigma_{h o m}$, is used as an estimate for the extinction coefficient $\sigma(r)$. Estimating the extinction coefficient allows us to perform a firstorder correction by removing the atmospheric transmittance from the NRB. As a result, we are able to obtain an approximation to the backscatter coefficient that can be linked directly to the aerosol number density given that the molecular contribution is insignificant as it generally is within the boundary layer where aerosols prevail.

Please note that using $\sigma_{\text {hom }}$ as an estimate for the extinction coefficient $\sigma(r)$ at all ranges introduces error particularly in regions where inhomogeneities occur. Nevertheless, we found the simplicity and stability of this approach to yield the best results. The solution proposed by Fernald [17] was considered as well, however, difficulties were encountered since the results produced were 
affected by inaccuracies on the far-end condition and error propagation while performing backward integration.

\section{RESULTS}

\subsection{Emissions}

The local utility companies in New York City, operate several peaking power generation stations throughout the city in which electricity is generated using fossil fuels (mostly natural gas). These plants are used to meet peak electric demands, especially during heat-wave days where air-conditioning electric loads exceed the power transported to New York City by long distance transmission lines. When the plants are in operation, they contribute to local emissions and resulting pollution within the City.

Our location at the City College of the City University of New York, is within $7 \mathrm{~km}$ of a number of such plants located in South Bronx and the Astoria Neighborhood in the Queens borough of New York City. In order to observe and capture emissions resulting from the generation of electricity taking place at these stations, continuous horizontal scans were carried out between the azimuth angles of $134^{\circ}$ to $141^{\circ}$ with respect to north, during daytime hours on July $1^{\text {st }}, 2018$. From these measurements, backscatter coefficient values were obtained as it was explained in section 2 . Figure 1 shows a map in which the backscatter coefficient values obtained from one of the scans are displayed. 4 different sources of emissions can be clearly identified within this narrow field of view. One of them is located in South Bronx at about $3.5 \mathrm{~km}$ away from our position. Two more are found $5 \mathrm{~km}$ away in the neighborhood of Astoria, Queens. And the last one, at $6.2 \mathrm{~km}$, is also located in Astoria, Queens. Monitored emissions are used to estimate plant activity during heatwave and non-heatwave days.

\subsection{Spatial Distribution}

Low income neighborhoods tend to have higher concentrations of vulnerable population to air pollution and often are in the vicinity of emission point-source and area sources [16]. A considerable amount of these neighborhoods are located in the Bronx borough of New York City which makes it an area of great interest for air pollution studies. On
May $10^{\text {th }}, 2017$, measurements over the Bronx area were taken for up to $4 \mathrm{~km}$ from our location between $40^{\circ}$ and $80^{\circ}$ azimuth angles with respect to north. A map with backscatter coefficient values from one of the scans is shown on Figure 2. In this particular case, a high load of aerosols is observed to concentrate over an area that corresponds to central Bronx.

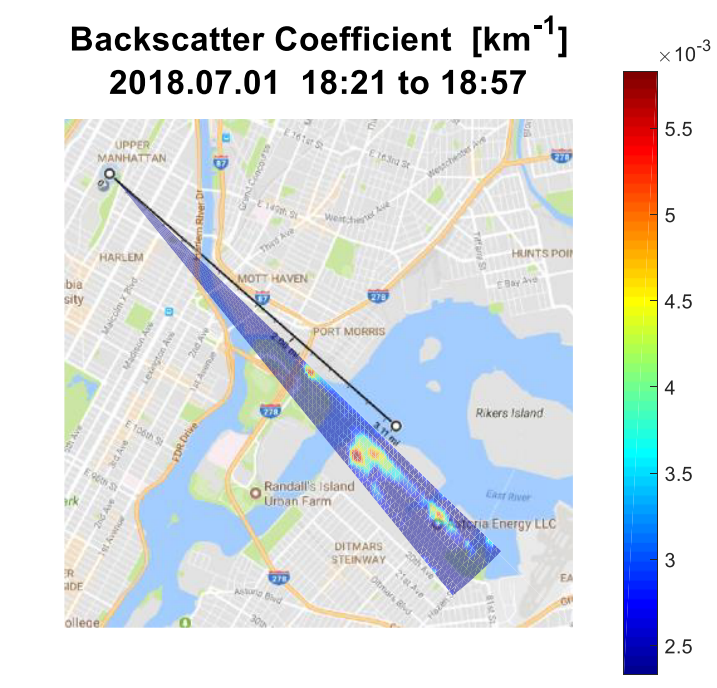

Figure 1. Backscatter coefficient map for July 1st, 2018 between 18:21 and 18:57 EDT.

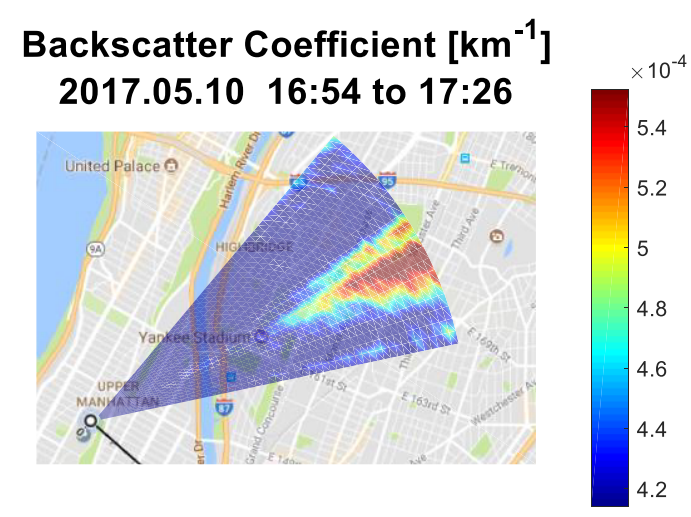

Figure 2. Backscatter coefficient map for May 10th, 2017 between 16:54 and 17:26 EDT.

\section{CONCLUSIONS}

Aerosol spatial distribution maps have been derived by applying the slope method to horizontal measurements taken with an eye-safe scanning MPL. Using the backscatter coefficient to represent the aerosol distribution provides an important improvement in terms of accuracy since far-end measurements can be better analyzed once the 
attenuation term is removed. Two case studies are shown looking at different regions within New York City. In both cases, measurements on the far end are of great importance as they show emissions and high concentrations of aerosols.

\section{ACKNOWLEDGEMENTS}

This study is supported by The National Oceanic and Atmospheric Administration - Cooperative Science Center for Earth System Sciences and Remote Sensing Technologies (NOAA-CESSRST) under the Cooperative Agreement Grant \#: NA16SEC4810008, and by the New York State Energy Research and Development Authority (NYSERDA) Agreement No. 100415. Its contents are solely the responsibility of the authors and do not necessarily represent the official views of the funding agencies.

\section{REFERENCES}

[1] C. A. Pope III, et al. Journal of the air \& waste management association 56(6), 709-742 (2006).

[2] S. A. Weber, et al. Environmental Research 151, 399-409 (2016).

[3] C. Gan, et al. Atmospheric Environment 45 (37), 6613-6621 (2011).

[4] Y. Wu, et al. Atmospheric Environment 63, 32-42 (2012).

[5] Y. Wu, et al. Atmospheric Environment 106, 358368 (2015).

[6] A. Behrendt, et al. Atmospheric environment, 45(16), 2795-2802 (2011).

[7] F. Späth, et al. Atmospheric Measurement Techniques, 9(4), 1701-1720 (2016).

[8] S. F. De Wekker, et al. Journal of Applied Meteorology and Climatology, 48(7), 1483-1499 (2009). [9] S.D. Mayor, et al. Optical Engineering, 46(9), 096201-096201 (2007).

[10] American National Standard for Safe Use of Lasers Outdoors, American National Standards Institute, New York (2015).

[11] J. D. Spinhirne, et al. IEEE Transactions on Geoscience and Remote Sensing, 31(1), 48-55. (1993).

[12] E. J. Welton, et al. Second International AsiaPacific Symposium on Remote Sensing of the Atmosphere, Environment, and Space, 151-158 (2001). [13] J. R. Campbell, et al. Journal of Atmospheric and Oceanic Technology 19(4), 431-442 (2002).

[14] J. D. Klett, et al. Applied optics, 20(2), 211-220 (1981).

[15] T. Dreischuh, et al. Aerosols-Science and Case Studies (2016).

[16] I. Kheirbek, et al. The New York City Community
Air Survey: neighborhood air quality 2008-2014 (2017). [14] F. G. Fernald, Applied optics, 23(5), 652-653 (1984). 